\title{
Multihop Wireless Channel Models suitable for Stochastic Petri Nets and Markov State Analysis
}

\author{
Rainer Schoenen ${ }^{1,2}$, Mohamed Rashad Salem ${ }^{2}$, Akram Bin Sediq ${ }^{2}$, Halim Yanikomeroglu ${ }^{2}$ \\ ${ }^{1}$ Communication Networks (ComNets), Faculty 6, RWTH Aachen University, Germany \\ ${ }^{2}$ Department of Systems and Computer Engineering, Carleton University, Canada \\ rs@comnets.rwth-aachen.de, halim@sce.carleton.ca
}

\begin{abstract}
In this paper the system analysis of modern wireless systems is simplified by providing simple yet powerful models for the wireless channel in the environment of higher layer abstract system descriptions with generalized stochastic Petri nets (SPN). This modeling approach is capable of deriving performance metrics in terms of packet delays even under heterogeneous, asymmetric, bursty and underutilized traffic conditions, because they are easy to model with SPN. The missing link in wireless systems are suitable channel models, which can now be used as a plug-in submodel inside a larger composite Petri net model. A number of models are proposed, starting from the finitestate Markov channel model approach. Performance results for a multihop relayed transmission under varied traffic load show the utility of this modeling approach.
\end{abstract}

Index Terms-stochastic Petri nets, channel models, Markov channel, relay, multihop

\section{INTRODUCTION}

$\mathbf{T}$ HE nature of wireless systems is dominated by the channel between antennas and channel characteristics are subject to complex phenomena. Pathloss, fading, shadowing, multipath propagation and multipoint to multipoint transmission are effects and dimensions engineers have to take into account in the physical layer models. On the top end of the ISO/OSI model, the performance is evaluated by terms of QoS contract satisfaction under diverse traffic conditions. Undoubtedly there is a huge gap in the theoretic support for all layers in one model. System level analysis therefore is only realistic if a simulation based approach is taken, where all effects and algorithms are implemented in software [1].

The medium access control (MAC) layer bridges the physical withe the higher layers, and allows a cross layer extension where required [2]. On the upper service access points to the MAC layer, the packet delay is already reasonably usable as performance measure for UDP flows [3].

In need for a theoretic system model to capture physical layer effects abstract enough for higher layers, Petri nets [4] are a promising way. Their advantage is the combination of the flexibility of a Turing-complete automaton with the power of stochastic Markov chain analysis. Stochastic Petri nets (SPN) and generalized SPN (GSPN) [5] have become a useful tool for adept researches in computer science. Results are obtained by numeric tools and do not require simulation. Useful tools for GSPN analysis exist [6]. In recent years, GSPN have been used occasionally to model communications systems [7], [8], but a widespread use is not observed, mainly due to fact that
SPN are unknown to most wireless researchers, unaware that Markov chains are a subset of SPN.

A number of promising SPN approaches already exist in all fields of wireless communications, e.g., resource management in cellular wireless systems [9], multihop (relay) transmissions [10], ad-hoc networks [11] and concrete technologies like IEEE 802.16 [12], IEEE 802.11 [13]. A simple twostate failure/recovery DSPN model for the GSM channel has been proposed in [14]. Links to higher layers exist in works for TCP models [15]. And there is still a high demand for recent technologies like LTE [16] and other IMT-Advanced systems [17]. Especially ISO/OSI layer two (medium access control) can benefit from GSPN analysis methods. The importance of packet delay aware models for resource and packet scheduling [18] and radio resource management [19] is obvious. Most works study the maximum throughput only with a full buffer assumption, because their methodology is unable to model traffic. Therefore these studies assume the overload condition and cannot provide finite delay results.

In this paper a link to the physical layer is introduced, by modeling the radio channel in a way suitable to be used as a building block in larger composite Petri nets. Several approaches are presented, but they basically fit into the scheme of finite-state Markov channels [20].

The paper is organized as follows: Section II introduces the basics of stochastic Petri nets, followed by the section on suitable channel models. Wireless system models based on this channel models are treated in section IV] Performance results are presented in section $\mathrm{V}$ before ending with the conclusion.

\section{Petri Nets}

Petri nets (PN) is a graphical and mathematical tool suitable to model complex systems with a state. Systems can be be described and studied when they are concurrent, asynchronous, distributed, deterministic or stochastic. Subclasses of $\mathrm{PN}$ are finite state machines and marked graphs used for DSP algorithms [21]. Many aspects of flow charts and description languages can be modeled with PN. There is plenty of literature on the underlying graph theory, liveness analysis, reachability set and other properties [4]. A PN is defined as a directed, weighted, bipartite graph having two sets of nodes called places $\left(P_{i}\right)$ and transitions $\left(T_{j}\right)$. Places are drawn as circles, transitions as boxes. Input arcs connect certain $P_{i}$ to $T_{j}$ with weight $w_{i j}$, output arcs connect $T_{j}$ to $P_{i}$ with multiplicity 
$v_{i j}$. Together they form the incidence matrix $\mathbf{D}=\left[v_{i j}-w_{i j}\right]$ When a PN consists of $M$ transitions and $N$ places, $\mathbf{D}$ is a $M \times N$ matrix (m rows, $\mathrm{n}$ columns). Places can contain an integer number of tokens (dots), all of which constitute the state called marking $\vec{m}$. The initial marking $\vec{m}_{0}$ is the start state. The notation $\# P_{i}=m_{i}$ means the current number of tokens in place $P_{i}$. An atomic action is the firing of a transition $T_{j}$ which changes the marking to

$$
\vec{m}_{k}=\vec{m}_{k-1}+\vec{t}_{k-1} \cdot \mathbf{D}
$$

with the firing vector $\vec{t}_{k-1}$ which is all zero except a single ' 1 ' at the j.th index. For further details of firing rules, disabling arcs, liveness and reachability see [4].

\section{A. Stochastic Petri Nets}

Stochastic PN (SPN) extend the paradigm to model time, essentially by assigning each transition a firing rate (in the continuous time case (CT)) or a firing probability (in the discrete time case (DT)). Here we will focus on the CT case only. Then the firing rates are given by $\vec{\Lambda}=\lambda_{1}, \ldots, \lambda_{m}$ which can be marking-dependent. $\lambda_{j}$ is the inverse of the average firing time $t_{j}$. Firing times are exponentially distributed, therefore memoryless, and the resulting reachability graph (RG) forms a Markov chain (MC) [5]. All arcs in the RG are annotated with the $\lambda_{j}$ of the transition $T_{j}$ responsible for the state change. The matrix $\mathbf{R}$ of all arc rates can then be used to calculate the steady-state solution of the MC and from that all other performance metrics can be obtained.

Generalized SPN (GSPN) combine both immediate (thick bar) and timed transitions (empty box), which also reduce to MC after condensing all tangible states, but offer a much higher modeling power. Priorities and weights can easily be annotated to immediate transition to model the outcome likelihood of deterministic and random decisions. Deterministic and stochastic SPN (DSPN) also allow one enabled transition with deterministic (fixed) firing time, denoted with a filled box.

In this paper we will not use colored PNs (CPN), as each token color extends the state space by huge amounts and an analytic/numeric treatment is often impossible. There is decent tool support for SPN and the MC is automatically determined and solved [6]. When the state space for numeric matrix solvers exceeds the current limit of around $10^{6}$ states on a standard PC, simulation can be used, based on an exhaustive repetition of the token game.

Queueing models and networks are a subset of the modeling power of SPN, and results for queue length and waiting times can easily be derived [22], that is why SPNs are becoming increasingly popular for modeling communication networks [7]. SPN can be used as well for modeling packet level and flow level, where a constant fluid of data in bits $/ \mathrm{s}$ is assumed while being in a state.

\section{CHANNEl MOdELS FOR SPN ANALYSIS}

Wireless channels possess a number of properties and are influenced by many effects. Usually they are decomposed into antenna gain, pathloss, slow fading, fast fading, shadowing, multipath propagation effects, which add up in the $\mathrm{dB}$ domain.

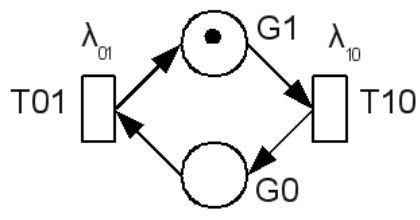

Fig. 1: SPN of the Gilbert-Elliot channel. The state \#G1 can be used in state-dependent formulations of SPN properties.

In this approach, only fading is considered frequency selective and time varying. Shadowing is location dependent and correlated [23], but without mobility it can be assumed constant. At the receiver, after procession of all the stages above, signal power and interference plus noise result in the signal-tonoise+interference (SINR) ratio. Depending on the modulation\&coding scheme used (with adaptive modulation\&coding, $\mathrm{AMC}$ ), in information theoretic sense the channel can carry a characteristic certain data rate given as mutual information (MI) in $b i t / s / H z$. Its capacity per antenna is limited by the Shannon bound. Depending on SINR and phymode bit errors result due to imperfect detection and residual errors after decoding. CRC detects packet errors and on its output the packet error ratio (PER) can be measured. Hybrid ARQ and packet ARQ blocks on layer 2 care for the retransmission of erroneous packets. More effects come into it by packet scheduling, finite queues and TCP flow control, but this is beyond the scope of channel models.

The simplest model suitable in a packet level SPN approach is the two-state Gilbert-Elliot (GE) channel, also seen as on-off MC. Its SPN equivalent is shown in Figure 1 using places $G_{1}$ and $G_{0}$, the token position in which marks the state as 'good' or 'bad'. The transition firing rates $\lambda_{10}$ (into fading) and $\lambda_{01}$ (recovery) model the state changes from 'good' to 'bad' and opposite. They are translated from the common parameters $P_{\text {error }}$ and $T_{\text {period }}$ by Eq. 2,3

$$
\begin{gathered}
\lambda_{10}=\left(T_{\text {period }} \cdot\left(1-P_{\text {error }}\right)\right)^{-1} \\
\lambda_{01}=\left(T_{\text {period }} \cdot P_{\text {error }}\right)^{-1}
\end{gathered}
$$

The state within itself can the be used in another part of the SPN to switch between transmitting or deleting packets which are currently transmitted on the channel.

The next level of complexity is the finite-state Markov channel (FSMC) [20]. It allows possible transitions between neighbor states with exponentially distributed timing of arbitrary mean value. Its $K$ states can model $K$ different receive conditions. Usually being in a state $k \in\{0,1, \ldots, K-1\}$ means having an SINR of $S$ in the interval $\left[S_{k}, S_{k+1}\right)$. The error probability is then a function of the $S_{k}$ in the center of the interval.

$$
S_{k}=\frac{S_{k}+S_{k+1}}{2}
$$

For the Rayleigh fading channel the SINR $S$ can be assumed exponentially distributed [20] with pdf $p_{S}(\sigma)$ and $\rho=E[S]$.

$$
p_{S}(\sigma)=\rho^{-1} \cdot \exp \left(-\sigma \rho^{-1}\right)
$$

The mobility speed at the receiver leads to the Doppler effect which is responsible for the rate at which state changes happen. Crossover probabilities and rates can be calculated 


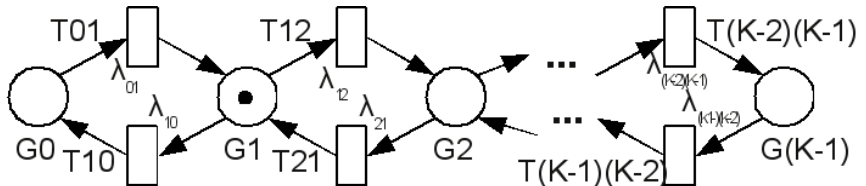

Fig. 2: SPN of the finite-state Markov channel. Explicit version. The token location defines the state.

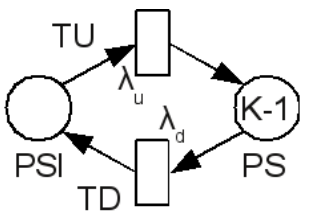

Fig. 3: SPN of the finite-state Markov channel. Condensed version. There can be $0 . .(K-1)$ token in each of the places.

from this, so that finally the Markov transition rates $\lambda_{i j}$ can be determined [20], which leads to the SPN transition firing rates in Figure 2 of the same value. Like in the GE channel, the channel state $C$ itself will be used to modulate (influence) a property of another SPN subnet, either as error probability $\epsilon=f(C)$, or as step to indicate the suitable phymode, when an AMC scheme is used and channel quality indication (CQI) is assumed accurate. The phymode then modulates the data rate of the wireless system during the time the channel is in this state.

Figure 3 shows a condensed version of the FSMC. Here the number of tokens in Place $P_{S}$ define the current state ( $K$ states altogether). Any dependent SPN structure is simply defined as a function of $\# P_{S}$. The firing rates $\lambda_{u}$ and $\lambda_{d}$ for the transitions $T_{u}$ going up and $T_{d}$ going down must be defined marking-dependent as

$$
\begin{gathered}
\lambda_{u}\left(\# P_{S}\right)=\sum_{k=0}^{K-2} \lambda_{k, k+1} \cdot\left(\# P_{S}=k\right) \\
\lambda_{d}\left(\# P_{S}\right)=\sum_{k=0}^{K-2} \lambda_{k+1, k} \cdot\left(\# P_{S}=k+1\right)
\end{gathered}
$$

where $\left(\# P_{S}=k\right)$ is the boolean operator $\left(\left(\# P_{S}=k\right)=1\right.$ if $\# P_{S}=k$ and 0 else). State dependent definitions in SPN are allowed for all properties that are resolved at the time of the creation of the RG. So it can be a transition firing rate, weight and priority (integer, i) of an immediate transition, the multiplicity (i) of an arc, etc.

The model before is limited to slow to medium fading, because transitions only occur between neighboring states. To allow for a more rapid change, the full incidence matrix $\mathbf{D}$ of this subnet can be utilized (full meshed PN), while the model before had only two nonzero entries per row in $\mathbf{D}$. The transition rated can be calculated by accumulating the transition frequency from one interval to another in a discretetime sampled system, which is easiest by means of simulation.

If an uncorrelated fading model is sufficient, then Figure 4 provides an SPN solution. The probability of each level is easily controlled by the weights of the immediate transitions following $P_{i}$ while the change rate is determined by $T_{\text {clock }}$.

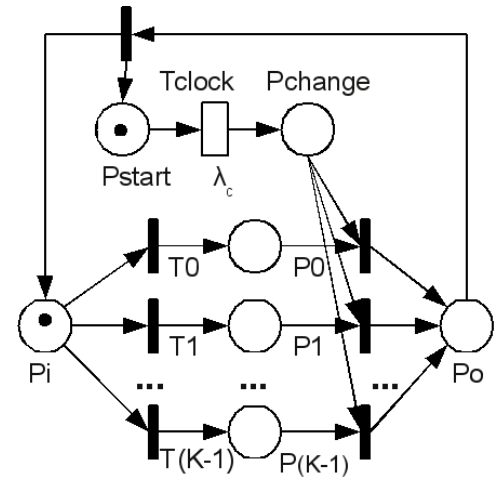

Fig. 4: SPN of uncorrelated fading

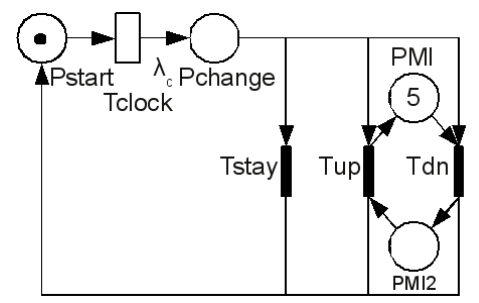

Fig. 5: SPN for modeling the distribution of phymodes

If only the phymode distribution on a channel is of interest, e.g. in order to model the capacity of a link (MI), the model in Figure 5 is convenient. It is similar to Figure 3 in that the weights of the transitions $T_{u p}$ and $T_{d n}$ must be defined marking-dependent, as a function of $\# P_{M I}$.

When the change of one phymode to another depends on the previous phymode (correlated model), then Figure 6 provides a way to keep track of the previous state, in order to calculate the transition weights in $T 0$ to $T 5$ which determine the probability of each phymode.

It is currently considered hard to model a frequency selective OFDMA channel, not because of the correlation of neighbor phymodes (which can be modeled), but due to the complexity of the state space. If 100 subchannels in LTE each have their individual phymode (let's assume with 5 different levels), then the set of markings consists of $5^{100}$ states, which is intractable by any MC solver, and only simulation is possible. It is highly recommended to reduce such systems to fewer subchannels and phymode levels, which still allows interesting principal analysis of systems. SPN are invaluable for cases where the MC state space is bounded and the structural complexity cannot be expressed by simple multidimensional birth-death processes, like shown in the following section.

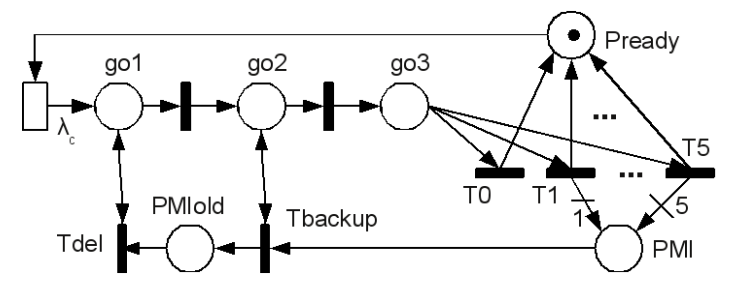

Fig. 6: SPN model to keep track of a previous state 

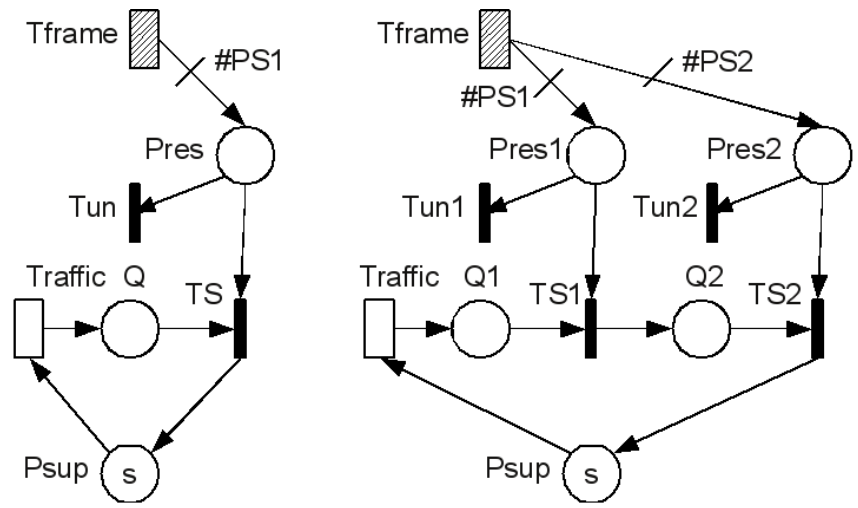

Fig. 7: SPN of a wireless system. Left: singlehop. Right: twohop relay transmission

\section{SPN MOdELS OF WiRELESS SySTEMS}

Wireless system models can be built using the channel models above by using the state (marking), i.e., the number of tokens in the characteristic places in the marking-dependent formulations of transition rates, weights, arc multiplicities (i) or priorities (i).

Figure 7 shows an example of such a system. It compares a singlehop wireless transmission (left) with a multihop transmission (right) in a relay-enhanced scenario like known from IMT-Advanced systems [17]. The channels are modeled as two separate subnets of type as in Figure 3, where the marking \#PS1 and \#PS2 define the multiplicity of the arc from the frame clock generator $\left(T_{\text {frame }}\right)$ to the container of available resources $P_{\text {res }}$. It produces a new bunch of resource blocks within the interarrival time of $\left.T_{\text {frame }} . T_{\text {frame }}\right)$ is a hatched box because we can allow an arbitrary timing. Here we use either an exponential interarrival time (for modeling random packet length of that kind) or deterministic constant time (for modeling constant-size packets and frame sizes). The traffic (packet) interarrival time is exponential, putting packets (tokens) into queue $Q$ or $Q 1$ correspondingly. In each frame the server $T_{S}$ can serve as many packets as there are resources for it in $P_{\text {res }}$. When $Q$ becomes empty before all resources in $P_{r e s}$ are used, they become 'unused' and will be cleared by $T_{u n}$, which has a lower priority than $T_{S}$. The supply place $P_{\text {sup }}$ is necessary to bound the state space. As a rule-of-thumb, the supply $s$ should be ten times larger than the expected queue occupancy in $Q$.

The model shown right of Figure 7 extends the single hop model with another wireless link, so that $T_{S 1}$ becomes the access on hop 1 and $T_{S 2}$ the relay access on hop 2. Both channels are independent, fading, but with the same average capacity. This way the performance of the multihop system can be analyzed and compared with the singlehop system. It also allows further studies, e.g., a flow control [24] to limit access to the relay packet buffer in $Q 2$. This is very useful, because one of the links (hop 1 or hop 2) is always a bottleneck, either in up- or downlink. For measurements the following equation derived from the p-invariant of the lower loop is useful:

$$
\begin{gathered}
\# Q 1+\# Q 2+\# P_{\text {sup }}=s \Rightarrow \\
E[\# Q 1+\# Q 2]=s-E\left[\# P_{\text {sup }}\right]
\end{gathered}
$$

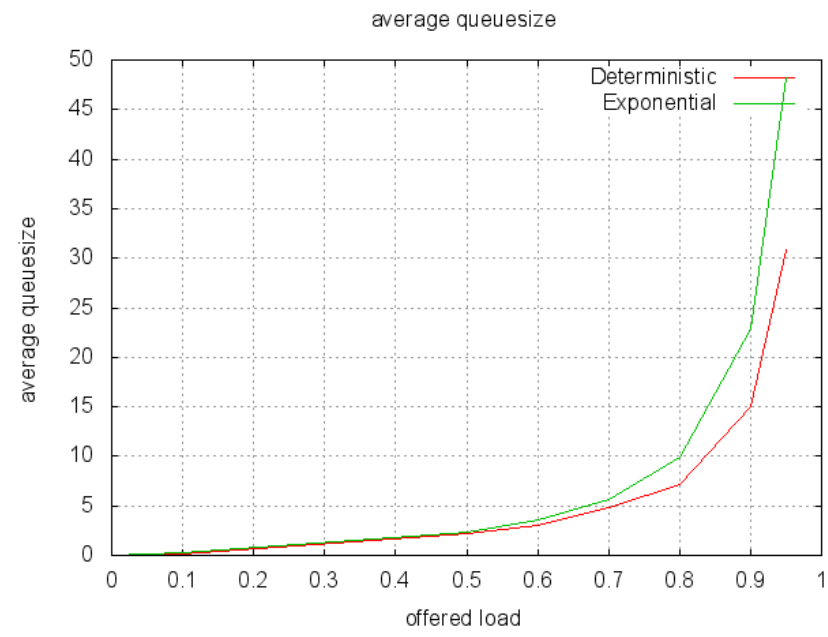

Fig. 8: Performance results of the SPN analysis of the singlehop system with deterministic (D) or exponential (M) firing times of $T_{\text {frame }}$.

In this model, priorities are in descending order with the intention to let hop 2 preempt first before new packets come in:

$$
\Pi\left\{T_{S 2}\right\}=4, \Pi\left\{T_{u n 2}\right\}=3, \Pi\left\{T_{S 1}\right\}=2, \Pi\left\{T_{u n 1}\right\}=1
$$

\section{Numeric Performance Results}

The system model has been analyzed by Markov chain analysis of the underlying MC (defined by the RG) of the SPN. The parameters used for this numeric example were: $K=3$ for three channel state levels with $M I_{0}=0, M I_{1}=1, M I_{2}=2$ representing the resource capacity proportional to bits $/ \mathrm{s} / \mathrm{Hz}$. The transitions were adjusted so that $P(\# P S=0)=5 \%$, $P(\# P S=1)=19 \%, P(\# P S=2)=86 \%$, i.e., with a $5 \%$ outage probability and a mean of $E[\# P S]=1.714$. The channel coherence time is chosen to be $T_{\text {period }}=10 \cdot T_{\text {frame }}$.

Figure 8 shows results for the study of different traffic arrival rates to the systems, normalized to the capacity of the system $(E[\# P S])$. The results reveal the queueing nature of random traffic with a server given by the wireless links. At a certain maximum load, the queue grows asymptotically to infinity, an unstable overload condition. We derive the necessity to introduce traffic separation into real-time and best effort by means of a static priority scheduler [25], which is also easy to model with SPN. Because all the MC state probabilities are known after analysis, it is easy to also obtain the probability density function (PDF) or CDF of the number of tokens in any place and therefore PDF and CDF of packet queue occupancy and packet delays.

Figure 9 shows results of the multihop model of Figure 7. with $T_{\text {frame }}$ as exponential. Without flow control, as analyzed here, the first hop performs the same as in the single- and multihop case, $E[\# Q 1]=E[\# Q]$. The second hop is almost identical to hop 1, which means that the traffic into $Q 2$ can also be assumed as memoryless (exponential interarrival time), because its queueing statistics are those of a $M / M / 1$ queue. 


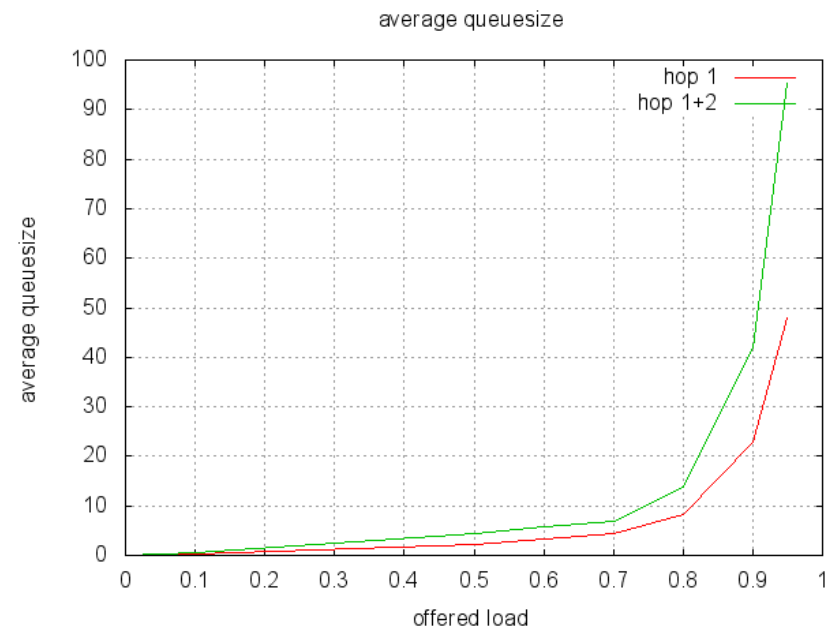

Fig. 9: Performance results of the SPN analysis of the multihop system compared to the singlehop system.

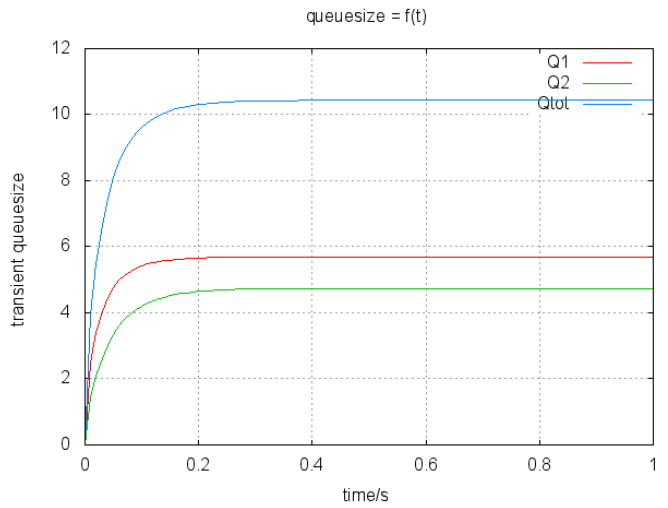

Fig. 10: Transient analysis of $E\left[\# Q_{1,2, t o t}(t)\right]=f(t)$.

Both hops together approximately perform with twice the delay of a singlehop system. This is not a big surprise, but it has been shown under channel conditions with outage and different phymode levels, i.e., an AMC situation common for all modern wireless networks. A transient analysis as shown in Figure 10 is easily to obtain by current tools and another benefit of this method.

\section{CONCLUSION}

A modeling approach using stochastic Petri nets (SPN) was proposed for studying the performance of wireless systems. The main contribution is the offer of different channel models which can readily be used as underlying Markov process for more complex structures built with SPN. As a result it becomes convenient to plug in one of these models and this enables to concentrate on the interesting algorithms on the MAC layer like resource allocation, scheduling, and the study of packet delays in wireless systems. Also important is the introduction of a static priority scheduler [25] and a reliable flow-control [24] for the bottlenecks in wireless systems. Future work will make more use of this and can provide analytic/numeric results where otherwise only simulation would have been used.

\section{REFERENCES}

[1] S. Max, D. Bültmann, R. Jennen, and M. Schinnenburg, "Evaluation of IMT-Advanced Scenarios Using the Open Wireless Network Simulator," in Proceedings of the 3rd International ICST Conference on Simulation Tools and Techniques (SIMUTools 2010), Mar 2010.

[2] R. Schoenen, "4G Cross-Layer Closed Loop Control Scheduling," in Proceedings of the 14th International OFDM Workshop (InOWo'09), Hamburg, Germany, Sep 2009.

[3] A. Otyakmaz, D. Bültmann, R. Schoenen, and I. Durmaz, "On Flow Management for Future Multi-Hop Mobile Radio Networks," in IEEE WiCom, Beijing, China, Sep 2009.

[4] T. Murata, "Petri Nets: Properties, Analysis and Applications," Proceedings of the IEEE, vol. 77, no. 4, pp. 541-581, April 1989.

[5] M. Marsan, Modelling with Generalized Stochastic Petri Nets. Wiley, 1996, iSBN 0-471-93059-8.

[6] R. German, "A toolkit for evaluating non-Markovian stochastic Petri nets," Performance Evaluation, vol. 24, pp. 69-87, 1995.

[7] J. Billington et al., Application of Petri Nets to Communication Networks. Springer, 1999, ISBN 3-540-65870-X.

[8] L. Lei, C. Lin, J. Cai, and X. Shen, "Performancs Analysis of Wireless Opportunistic Schedulers using Stochastic Petri Nets," IEEE Transactions on Wireless Communications, vol. 8, no. 4, April 2009.

[9] F. Jaimes-Romero, D. Munoz-Rodriguez, C. Molina, and H. Tawfik, "Modeling resource management in cellular systems using Petri nets," in IEEE Transactions on Vehicular technology, May 1997, pp. 298-312.

[10] R. Schoenen, "Unorthodox abstract models for the OFDMA multihop transmission," in Proceedings of the 13th International OFDM Workshop (InOWo'08), Hamburg, Germany, Aug 2008.

[11] C. Zhang and M. Zhou, "A stochastic Petri net-approach to modeling and analysis of ad hoc network," in Proceedings of the ITRE, Aug 2003.

[12] S. Geetha and R. Jayaparvathy, "Modeling and Analysis of bandwidth Allocation in IEEE 802.16 MAC: A Stochastic Reward net Approach," Int. J. Communications, Network ans System Sciences, vol. 3, no. 7, pp. 631-637, July 2010.

[13] R. Jayaparvathy, S. Anand, S. Dharmaraja, and S. Srikanth, "Performance Analysis of IEEE 802.11 DCF with Stochastic Reward Nets," in International Journal of Communication Systems, , vol. 20, no. 3, 2007.

[14] A. Zimmermann and G. Hommel, "A Train Control System Case Study in Model-based Real Time Systems Design," in Proceedings of the International Parallel and Distributed Processing Symposium (IPDPS'03), Apr 2003.

[15] R. Gaeta, M. Gribaudo, D. Manini, and M. Sereno, "On the use of Petri nets for the computation of completion time distribution for short TCP transfers," Proceedings of the 24th international conference on Applications and theory of Petri nets, vol. LNCS, pp. 181-200, 2003.

[16] R. Schoenen, Long Term Evolution. ISBN 978-1-4200-7210-5: Taylor and Francis - CRC Press, 2009, vol. 71, ch. Multihop Extensions to Cellular Networks - The Benefit of Relaying for LTE, pp. 265-303.

[17] J. Monserrat, P. Sroka, G. Auer, J. Cabrejas, D. Martin, A. Mihovska, R. Rossi, A. Saul, and R. Schoenen, "Advanced radio resource management for IMT-Advanced in WINNER+ (II)," in Proc. ICT-MobileSummit 2010, Florence, Italy, Jun 2010.

[18] R. Schoenen and F. Qin, "A Closed Loop Control Model separating Resource and Packet Scheduling in Multihop Cellular Networks," in Proc. of the European Wireless, Lucca, Italy, Apr 2010.

[19] M. Salem, A. Adinoyi, H. Yanikomeroglu, and D. Falconer, "Opportunities and challenges in OFDMA-based cellular relay networks: A radio resource management perspective," Vehicular Technology, IEEE Transactions on, June 2010.

[20] H. Wang and N. Moayeri, "Finite-State Markov Channel - A Useful Model for Radio Communication Channels," IEEE Transactions on Vehicular Technology, vol. 44, no. 1, pp. 163-171, Feb 1995.

[21] V. Živojnović, R. Schoenen, and H. Meyr, "On Retiming of Multirate DSP Algorithms," in Proceedings of the IEEE International Conference on Acoustics, Speech and Signal Processing, vol. VI, Atlanta, May 1996.

[22] L. Kleinrock, Queueing Systems, Vol. I: Theory. New York: John Wiley \& Sons, 1975.

[23] S. Szyszkowicz, H. Yanikomeroglu, and J. Thompson, "On the feasibility of wireless shadowing correlation models," IEEE Transactions on Vehicular Technology, Oct 2010.

[24] R. Schoenen, G. Post, and A. Müller, "Analysis and Dimensioning of Credit-Based Flow Control for the ABR Service in ATM Networks," in Proceedings of the IEEE GLOBECOM, 1998, vol.4 p.2399.

[25] R. Schoenen and A. Otyakmaz, "QoS and Flow Management for Future Multi-Hop Mobile Radio Networks," in IEEE VTC Fall, Ottawa, Canada, Sep 2010 . 